\title{
A CONCEPTUAL FRAMEWORK FOR VIRTUAL GEOGRAPHIC ENVIRONMENTS KNOWLEDGE ENGINEERING
}

\author{
Lan You ${ }^{\mathrm{a}, \mathrm{b}}$, Hui Lin ${ }^{\mathrm{a}, \mathrm{c}, *}$ \\ a Institute of Space and Earth Information Science, Chinese University of Hong Kong, Hong Kong, China - (youlan, \\ huilin)@cuhk.edu.hk \\ ${ }^{\mathrm{b}}$ Faculty of Computer Science and Information Engineering, Hubei University, Wuhan, China \\ ${ }^{c}$ Department of Geography and Resource Management, Chinese University of Hong Kong, Hong Kong, China
}

Commission II, WG II/5

KEY WORDS: Geographic knowledge, Knowledge engineering, VGE, Framework, Knowledge evolution

\begin{abstract}
:
VGE geographic knowledge refers to the abstract and repeatable geo-information which is related to the geo-science problem, geographical phenomena and geographical laws supported by VGE. That includes expert experiences, evolution rule, simulation processes and prediction results in VGE. This paper proposes a conceptual framework for VGE knowledge engineering in order to effectively manage and use geographic knowledge in VGE. Our approach relies on previous well established theories on knowledge engineering and VGE. The main contribution of this report is following: (1) The concepts of VGE knowledge and VGE knowledge engineering which are defined clearly; (2) features about VGE knowledge different with common knowledge; (3) geographic knowledge evolution process that help users rapidly acquire knowledge in VGE; and (4) a conceptual framework for VGE knowledge engineering providing the supporting methodologies system for building an intelligent VGE. This conceptual framework systematically describes the related VGE knowledge theories and key technologies. That will promote the rapid transformation from geodata to geographic knowledge, and furtherly reduce the gap between the data explosion and knowledge absence.
\end{abstract}

\section{INTRODUCTION}

Geographic knowledge is very important for the virtual geographic environments (VGE), since it helps to simulate and predict the earth science mechanism processes (Laurini, 2014; Lin et al., 2013). With the rapid increase of earth observation technique and serious absence of the information value-added mechanism, the gap between the data explosion and knowledge absence has been a common phenomenon in research area. Utilizing multi-dimensioned representation and deep analysis model on geo-science problems, VGE can promote the rapid transformation from geodata to geo-knowledge (Li et al., 2002). Studies on geographic knowledge engineering in VGE are both urgent and important.

The knowledge is a kind of structural, interconnected, and everincreasing information, which derived from the interaction with people as well as the analysis and application of the relationships and laws mined by other assistant technology (Maurer, 1999). The geographic knowledge is the extension of the knowledge connotation in geoscience realm. ESRI thinks the geographic knowledge is a universal knowledge concept related to the geo-location, which includes geodata, geo-model, metadata, geoprocessing workflow and cartographic representations (Dangermond, 2010). Through explaining and solving the geographic science problems, the geographic knowledge can be acquired as a useful geo-information (Huang, 2008). The geographic knowledge refers to the geo-information used to resolve the geoscience problems existing in different research realms such as archaeology, ecology, botany and so on(Kim et al., 2012). From the aspects of the spatial cognition and knowledge sharing, some researches have been done around the geographic knowledge theory and methods (Wang et al., 2005; Gong et al., 2014). According to the limitation problem in the access and search of the structural knowledge base, a knowledge management method based on social network was proposed (Von Krogh, 2012). Some researches based on social mining were further done about the knowledge obtaining and management (Colombo et al., 2011; Alonso et al., 2013). How to draw the geographic knowledge and attributes data from the remote sensing images has been researched (Aldridge, 2001; Crowther, 1999). Some researchers (Ester et al., 1997; Mennis et al., 2003; Miller et al., 2009) studied the methods of discover and extraction the geographical knowledge through spatial mining. Ontology is always used to represent the knowledge (Karalopoulos et al., 2005; Teller et al., 2007) as well as XML (Mani et al., 2010). The essence of the geographic knowledge has been described from three aspects: thought, reasoning and reuse (Golledge, 2002). According to the structure of knowledge body, a computation framework of geographic information technique knowledge body (GIS\&T BoK) was proposed (Ahearn et al., 2013).

The above researches focus only on the knowledge in the traditional GIS realm. Some scholar proposed an Informed Virtual Geographic Environments (IVGE) to support the geographical process simulation based on multi-agents (Mekni et al., 2011), which talked about the representation and reasoning of the geographic knowledge in VGE.

Apparent with the traditional GIS, VGE process models have the features of cross-disciplinary, multi-interaction, multidimension and intensive data or computation. Those cause VGE having their own geographic knowledge architecture and

\footnotetext{
* Corresponding author. Email: huilin@ cuhk.edu.hk
} 
characteristics. However, most of the existing researches around the geographic knowledge focus on the traditional GIS realm and are not described and understood systematically.

\section{KNOWLEDGE IN VIRTUAL GEOGRAPHIC ENVIRONMENTS}

\subsection{VGE Knowledge (VGE-K)}

Geographic knowledge refers to the useful geo-information which explains and solves the geographic science problems. It is the extension of the knowledge concept in geographic science realm. Apparent with traditional GIS analysis, VGE focuses more on multi-resources geodata integration, geo-science problem analysis, geographic law mining and spatial-temporal phenomenon simulation by utilizing multi-dimensional sensing technique and earth mechanism models (Lü, 2011). In virtual geographical environment, different forms of geographic knowledge from different research realm can be fused for knowledge innovation. That not only changes the acquisition, representation and update methods of the traditional GIS knowledge but also promotes the multidisciplinary thinking collision helping to generate the new knowledge.

The VGE knowledge should be the related geo-information used to solve some kind of geo-science problem, explain some earth phenomena and draw some geographic rules through VGE technologies which is always abstracted and repeatable. They may be the experts' experiences in geographic research realm, deduction process of the model building, the basic GIS theories, the geoprocessing value-added results and etc.

\subsection{Features of VGE-K}

During the whole analysis process in VGE, VGE-K is present as different forms which gradually evolve into the explanations for the geo-science problems. VGE-K is the extending of the geographic knowledge connotation in VGE which help to build an intelligent and high efficient VGE framework. It is abstract, repeatable and renewable. In addition, it has the following typical features:

1. Earth mechanism process relevance

2. Time span relevance

3. Space span relevance

4. Multi-disciplinary relevance

5. Details levels relevance

6. Multi-factor relevance

7. Uncertainty

Earth mechanism model is the core of VGE supporting the spatiotemporal process simulation whereas GIS analysis relies on the geodata processing model. VGE can present the evolving process of the earth mechanism since they deal with geodata across long time and wide space. Aiming at answering some geo-science problem, crossing-disciplinary knowledge is needed generally such as land use, population, policy, oceanology, and so on. The complexities of earth mechanism caused the multifactor dependency of the VGE process as well as the uncertainty.

\subsection{Evolution process}

The knowledge in VGE has an evolution process covering knowledge modelling, knowledge collaboration, knowledge innovation, and knowledge sharing stages. In knowledge modelling stage, VGE-K model is built for answering some geo-science problem. Then knowledge from different research realm is fused to work in one VGE-K model whose conclusion maybe the answer of the problem. The 3rd stage is the adjustment and optimization stage during which the researchers' cognition can be used. The 3rd stage can go back to the 2nd stage when the VGE-K model needs to be updated. Once the new VGE-K is approved to be useful, it can be shared and spread.

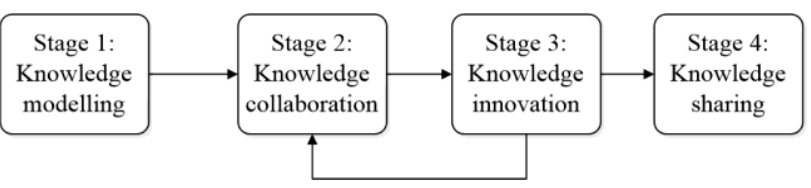

Figure 1. VGE-K evolution process

\subsection{VGE-K Engineering}

From the perspective of knowledge engineering, VGE-K engineering should be the theoretical and technical system for VGE-K's management. VGE-K engineering is the interdisciplinary science of knowledge engineering and VGE, which offers a support for the complex geo-science problems. VGE-K engineering will provide the theory and technique basis to construct an intelligent VGE aiming to speed up the transformation process from geodata to geo-knowledge.

\section{A CONCEPTUAL FRAMEWORK FOR VGE KNOWLEDGE ENGINEERING}

\subsection{Conceptual Framework Model}

This paper proposes a conceptual framework model which presents a theoretical and technical system for the VGE knowledge engineering. As figure 2 shown, the framework model contains six parts around VGE-K: representation \& modelling, management, collaboration \& evolution, visualization, evaluation and sharing and spread.

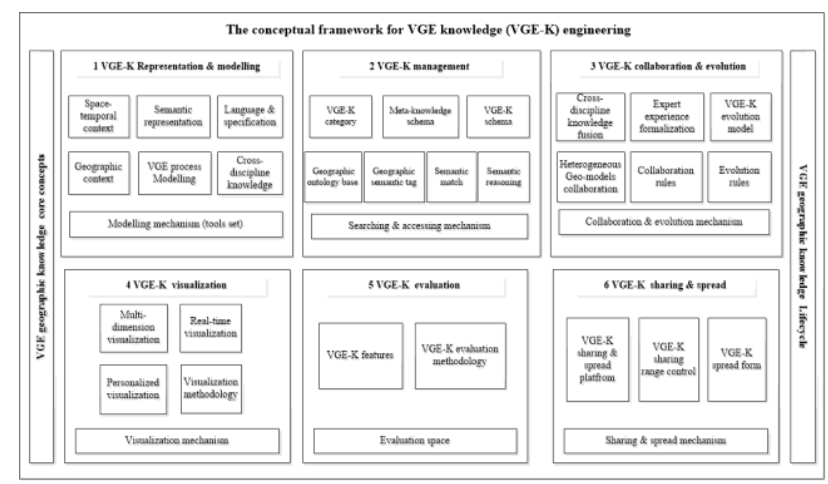

Figure 2. The conceptual framework model for VGE-K

$$
\text { engineering }
$$

\subsection{Representation and modelling}

How to represent the geo-knowledge is the top priority in VGE$\mathrm{K}$ engineering. To fully describe the VGE-K, the following aspects should be contained: geo-context, geoprocessing business logic, space-temporal deduction process, metadata, semantic descriptions and involved cross-discipline knowledge. The VGE-K modelling mechanism should be researched to create a new VGE-K model easily as well as the corresponding tool. A representation specification is needed to support the VGE-K model building as the model description language. 


\subsection{VGE-K management}

The VGE-K management involves in the store and organization of the geographic knowledge determining whether users search, use and share it fast and accurately. Through constructing a VGE-K base, the geo-knowledge can be searched, organized, integrated, reasoned and shared. The knowledge classification is the basis of the organizational structure of the VGE-K base. The meta-knowledge is the abstract description of the VGE-K. Each VGE-K model is identified with some semantic tags representing its function in essence. By creating the geoknowledge ontology base VGE-K can be searched, matched and reasoned when users need to solve some geo-science problem.

\subsection{Collaboration and Evolution}

Multi-disciplinary knowledge is always required to work collaboratively in VGE analysis processes. The collaboration of knowledge from different research realms involves in experiences formalization, evolution process modelling, heterogeneous geo-models integration, and the constraint rules in the collaboration and evolution processes.

\subsection{Visualization}

Visualization is one of the core components of the VGE-K since dynamic multi-sensing technique is the typical feature of VGE. Different visualization methods should be used to present different VGE-K according different perspectives of profession background, spatiotemporal context, and description details level. A completed visualization methodology system for VGE$\mathrm{K}$ should be researched.

\subsection{Evaluation}

The evaluation methodology should be built to measure whether the VGE-K is available. The availability decides how easily VGE-K can be shared and how widely VGE-K can be spread. Building the evaluation methodology should be combined with the features of VGE-K. A well-established evaluation methodology will greatly promote the VGE-K's generation, sharing, and spread.

\subsection{Sharing and Spread}

A collaboration platform for sharing and spreading the VGE-K is required to contain the VGE-K models oriented to different geo-science problem. A uniform VGE-K description specification should be used for spreading various forms of VGE-K. Different researchers' backgrounds and tasks allocation should be used to limit the operations and controls scope of VGE-K which forms the collaboration rules.

\section{CONCLUSIONS}

Geographic knowledge is the useful geo-information explaining and solving the geo-science problems which is the knowledge concept extension in GIS realm. Compared with the traditional GIS, the VGE geographic knowledge is mainly oriented to the geo-science problem, geographical phenomena and geographical laws supported by VGE. Fewer existing researches were made in the VGE-K. This paper firstly analysed the features of VGE knowledge and clearly defined the VGE-K and VGE-K engineering. Then a conceptual framework for VGE knowledge engineering was proposed in order to effectively manage and use geographic knowledge in VGE. Combining with previous theories and technique on knowledge engineering and VGE, the VGE-K conceptual framework model is composed with six parts: representation \& modelling, management, collaboration and evolution, visualization, evaluation, and sharing and spread. This conceptual framework proposed the key problems and probable research directions providing the basic theory and methodology for an intelligent VGE in the near future. The proposed conceptual framework will contribute to the rapid transformation from geodata to geographic knowledge in VGE. And it will furtherly reduce the gap between the data explosion and knowledge absence.

\section{ACKNOWLEDGEMENTS}

This work was supported by China National key basic research development 973 projects [2015CB954103] and National Natural Science Foundation of China for young scholars [41401464].

\section{REFERENCES}

Ahearn, S., Icke, I., Datta, R., DeMers, M. N., Plewe, B., \& Skupin, A. 2013. Re-engineering the GIS\&T Body of Knowledge, International Journal of Geographical Information Science, 27(11), pp. 2227-2245.

Aldridge, C., 2001. Geographic Knowledge: Bringing Geographers to Their Sensors. In: Thirteenth Annual Colloquium of the Spatial Information Research Centre. PA Whigham (Ed), Vol. 2, No. 5, pp. 195-210.

Alonso, O., Banavar, H., Davis, M. E., \& Khandelwal, K. 2013. Knowledge discovery using collections of social information. In: Google Patents. No. 13/830, 944.

Colombo, M. G., Rabbiosi, L., \& Reichstein, T., 2011. Organizing for external knowledge sourcing. European Management Review, 8(3), pp. 111-116.

Crowther, P., 1999. A visual geographic knowledge classification and its relationship to the KADS model. In: Advanced Topics in Artificial Intelligence. Springer. Berlin Heidelberg. pp. 478-479.

Dangermond, J., 2010. "Geographic Knowledge: Our New Infrastructure",http://www.esri.com/news/arcnews/winter1011ar ticles/geographic-knowledge.html (28 Mar. 2016).

Ester, M., Kriegel, H. P., \& Sander, J. 1997. Spatial data mining: A database approach. In: Advances in spatial databases. Springer, Berlin, Heidelberg. pp. 47-66

Golledge, R. G., 2002. The nature of geographic knowledge. Annals of the Association of American Geographers, 92(1), pp. $1-14$.

Karalopoulos, A., Kokla, M., Kavouras, M., 2005. Comparing representations of geographic knowledge expressed as conceptual graphs. GeoSpatial Semantics. Springer, Berlin, Heidelberg. pp. 1-14.

Kim, T. J., Wiggins, L. L., Wright, J. R. (Eds.). 2012. Expert systems: Applications to urban planning. Springer Science \& Business Media.

Laurini, R., 2014. A conceptual framework for geographic knowledge engineering, Journal of Visual Languages \& Computing, 25(1), pp. 2-19. 
Lin, H., Chen, M., Lu, G., Zhu, Q., Gong, J., You, X., Wen, Y., $\mathrm{Xu}, \mathrm{B} ., \mathrm{Hu}, \mathrm{M} ., 2013$. Virtual geographic environments (VGEs): a new generation of geographic analysis tool. Earth-Science Reviews, 126, pp. 74-84.

Mani, I., Doran, C., Harris, D., Hitzeman, J., Quimby, R., Richer, J., Wellner, B., Mardis, S., Clancy, S., 2010. SpatialML: annotation scheme, resources, and evaluation, Language Resources and Evaluation, 44, pp. 263-80.

Maurer, H., 1999. The heart of the problem: Knowledge management and knowledge transfer. In: Enable, Vol. 99, pp. 8-17.

Mekni, M., Environnement, E., Moulin, B., 2011. Informed Virtual Geographic Environments for Knowledge Representation and Reasoning in Multiagent Geosimulations. Cognitive.

Mennis, J., Peuquet, D, 2003. The role of knowledge representation in geographic knowledge discovery: a case study. Transactions in GIS, 7(3), pp. 371-391.

Miller, H. J., Han, J., 2009. Geographic data mining and knowledge discovery. CRC Press.

Teller, J., Lee, J., Roussey, C., 2007. Springer, Ontologies for urban development.

Von Krogh, G., 2012. How does social software change knowledge management? Toward a strategic research agenda. The Journal of Strategic Information Systems, 21(2), pp. 154164.

Gong, J., Geng, J., Wu, H., 2014. Geospatial Knowledge Service: A Review, Geomatics and Information Science of Wuhan University, 39(8), pp. 883-890.

Huang, H., 2008. Research on key technologies of Geospatial Knowledge Service [D], Wuhan, Wuhan University.

Li, D., Wang, S., Shi, W., Wang, X., 2001. On spatial data mining and knowledge discovery. Geomatics and Information Science of Wuhan University, 26(6), pp. 491-499.

Lü, G., 2011. Geographic analysis-oriented virtual geographic environment: framework, structure and functions. Science China Earth Sciences, 54(5), pp. 733-743.

Wang X., Liu Y., Zhang J., 2005. Geo-Spatial Cognition: An Overview, Geography and Geographic Information Science, 21, pp. 1-10. 